\title{
Vigilancia Tecnológica: directriz para el éxito organizacional Descripción y contribuciones de una disciplina orientada a la eficiencia de las organizaciones de base tecnológica
}

José Humberto Robin ${ }^{1}$, Rosana Hadad Salomón ${ }^{1}$, Celeste Quiroga Hamoud $^{1}$ y Soledad Rasgido ${ }^{1}$

\section{Resumen}

La Vigilancia Tecnológica es parte de una disciplina que actualmente tiene protagonismo y una gran repercusión dentro de cualquier organización con enfoque de innovación, expansión y permanencia a través del tiempo. Abarca un conjunto de metodologías, herramientas y procedimientos con los cuales se identifican y reúnen datos de forma sistemática y organizada, que son analizados y transformados en información y conocimientos relevantes sobre las condiciones tanto del entorno de la organización como del ambiente interno, llegando a las personas correspondientes para definir y establecer decisiones estratégicas dentro de la organización.

Palabras Clave: Vigilancia Tecnológica - Conocimiento Industrial. 


\section{Abstract}

Technology Surveillance is part of a discipline that currently outstands and has a great impact in any organization focused on innovation, expansion and continuity. It encompasses a set of methodologies, tools and procedures which identify and collect data in a systematic and organized manner. Data is analysed and transformed into relevant information and knowledge of both environment and organization's internal environment conditions, reaching the appropriate people to define and establish strategic decisions within the organization.

Keywords: Technology Surveillance - Industrial Knowledge. 


\section{Introducción}

La Vigilancia Tecnológica (en adelante VT) se constituye en uno de los esfuerzos más importantes por parte de las organizaciones para captar, analizar y tomar decisiones con base en la información que éstas pueden adquirir proveniente del exterior, sustentados en la calidad, pertinencia y utilidad de la misma. Su uso, de manera similar a la Gestión del Conocimiento, ha sido adoptado por grandes organizaciones con miras a no ser sorprendidas tecnológicamente por sus competidores (Rodríguez, 1999).

La VT aparece como una de las funciones clave para la gestión de la tecnología por cuanto, para mantener en vanguardia una cartera tecnológica, es esencial la detección de los cambios y las nuevas tecnologías con la suficiente antelación para poder evaluarlas y prepararse, bien sea para su adopción y explotación o para entender las dinámicas del conocimiento en un área determinada (León López, A.; Castellanos Domínguez, O.; Montañez Franco, V., 2008). Se debe inspeccionar permanentemente el cuerpo de conocimientos científicos existentes; es necesario vigilar antes de emprender cualquier proyecto de innovación, con el objeto de no duplicar esfuerzos en hacer o lograr algo que ya existe y se encuentra disponible para su utilización.

La VT es una función continuada en el tiempo y muy ligada a los aspectos estratégicos de la empresa, es un estado permanente de atención y toma de decisiones ante oportunidades y amenazas del entorno. Conlleva labores de inteligencia por cuanto la información que se capte debe estar sustentada en las metas fijadas por los directivos de una organización, para que ésta se constituya, posteriormente, en el valor agregado de toda estrategia corporativa destinada a alcanzar el éxito.

\section{El Ciclo de la Vigilancia Tecnológica}

La puesta en marcha de procesos de Vigilancia Tecnológica consiste en la implementación de un sistema en el que a través de un conjunto de métodos, procedimientos y recursos, la información es sistemáticamente captada, analizada y difundida como inteligencia a los directivos de una organización, quienes pueden actuar a partir de ella. Este proceso se da gracias a la implementación del Ciclo de trabajo de la VT (figura 1), el cual tiene las siguientes características (Ministerio de Agricultura y Desarrollo Rural de Colombia, 2008): 


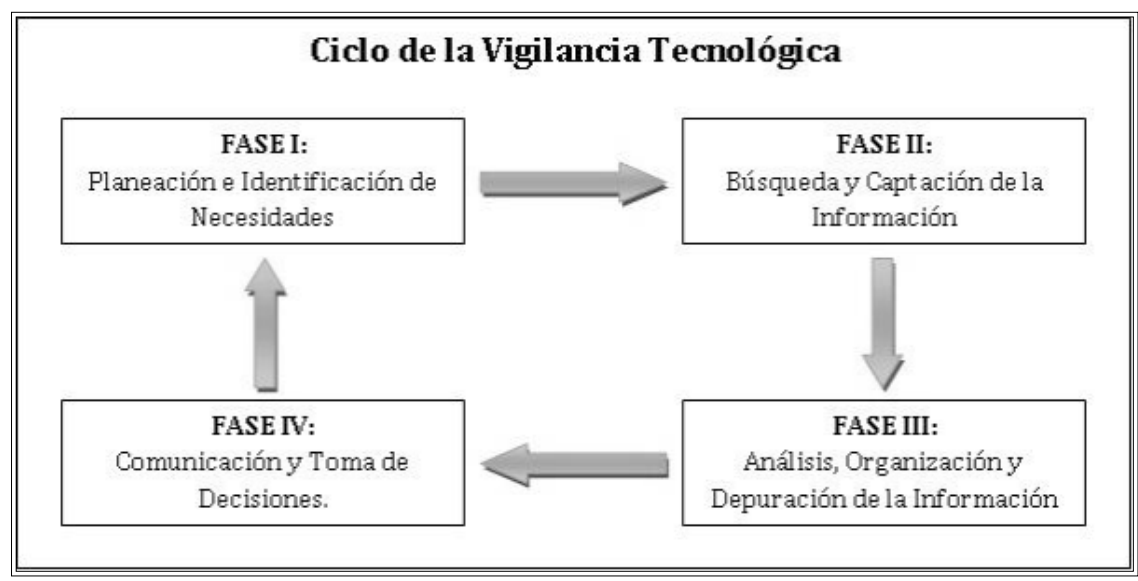

Figura 1. Ciclo de la Vigilancia Tecnológica y sus fases constitutivas.

A. Un ciclo fundamentalmente está compuesto por una serie de fases sucesivas entre sí: las dos primeras fases son de captación y organización de la información. Las dos últimas son de "inteligencia" para la toma de decisiones.

Esta sutil pero vital diferenciación lleva detrás un concepto fundamental: la cadena de transformación de la información. Es decir, en las primeras fases de captación se tienen los datos e incluso información con sentido general, mientras que en las segundas fases la información tiene un valor añadido que la convierte en conocimiento y que al ser utilizado para la toma de decisiones se convierte en Inteligencia.

B. Cada una de las fases del Ciclo de VT, a pesar de su secuencialidad, debe retroalimentar, interactuar y validar cada uno de sus resultados con el entorno y con los expertos.

C. En la primera fase de Planeación se identifican los Factores Críticos de la Vigilancia Tecnológica (en adelante FCVT), es decir, temáticas en las cuales se concentrará el trabajo. Los FCVT permiten dar al Ciclo las características de continuidad y focalización: continuidad en la medida en que los FCVT o las necesidades de hoy, no son obligatoriamente las necesidades del mañana y por ello se tiene un circuito de retroalimentación; y focalización porque es determinante realizar el monitoreo de riesgos y oportunidades estratégicas para garantizar la supervivencia de la organización.

D. El Ciclo de VT puede llevarse a cabo iterativa e infinitamente. Sin embargo, si este no tiene como objetivo incidir en el futuro de la organización y en la definición de sus estrategias, no tiene sentido realizar ninguna de las fases del ciclo.

E. Una vez que el Ciclo de VT se lleva a cabo por completo, es imprescindible iniciar con la etapa de comunicación. Los hallazgos no tienen sentido si no se difunden en la organización. Por lo tanto, es necesario identificar los canales de comunicación y la forma como se toman las decisiones en el interior de la organización. 


\section{La Vigilancia Tecnológica y las Herramientas de Software}

Reconociendo que la Vigilancia Tecnológica es un valioso recurso para las organizaciones que contribuye a obtener información útil y siempre actualizada en el ámbito de su incumbencia, también debemos reconocer que para llevar a cabo el proceso vinculado a esta disciplina, resulta imprescindible contar con herramientas de software que cooperen, en la medida en que se precise, con las etapas constitutivas del Ciclo de VT. Es inevitable la conjunción de la VT con aplicaciones informáticas ya que combinadas y orientadas hacia un objetivo común cobran una singular trascendencia que logra condensarse en un complemento capaz de apoyar la toma de decisiones en una estructura organizacional.

Es importante mencionar que la Vigilancia Tecnológica no insta a "acumular" herramientas de software por montón. Una organización jamás debe encaminarse en la búsqueda compulsiva de aplicaciones informáticas sin contar con un claro criterio de selección de las mismas de acuerdo a los objetivos planteados y considerando el alcance que tendrá el proceso de VT que se quiera llevar a cabo.

Por el contrario, la VT promueve la indagación y adquisición de aquellas herramientas que sirvan a los propósitos de la organización como medio a través del cual se ejecuten las fases de su Ciclo. La VT y las herramientas de software se encuentran íntimamente vinculadas, combinándose ambas y articulándose en conjunto para servir a las metas de captación e identificación de oportunidades y potenciales amenazas en el plano tecnológico, de manera de capitalizar esa información en decisiones inteligentes. Un buscador especializado, una base de datos inteligente, un gestor de datos para análisis estadísticos, son aplicaciones de gran utilidad que pueden aportar invaluables contribuciones que posteriormente se traduzcan en altos niveles de eficiencia, eficacia y rentabilidad.

\section{Sistema de VT: Prototipo de Vigilancia Tecnológica}

En la actualidad, las empresas pierden importantes cantidades de dinero al invertir en proyectos cuyo resultado ya se encuentra patentado. En la mayoría de los casos, el motivo del desconocimiento es debido a la situación provocada por el gran volumen de información existente, quedando de manifiesto la imperiosa necesidad de conocer y disponer de aquella información adecuada en el momento oportuno para una correcta toma de decisiones. En este contexto, estamos convencidos que un Sistema de Vigilancia Tecnológica se erige como una respuesta confiable y eficiente. 
Si bien son pocas las empresas que tienen el privilegio de ofertar sus productos sin competencia, existe una gran dependencia del entorno en el que se encuentran (competidores, proveedores, clientes) y la organización debe estar informada de los cambios y sus posibles repercusiones, por lo que el conocimiento del entorno pasa a ser una actividad crítica para su buen desempeño (Villanueva, M., 2010).

Sabemos que en la actividad de VT, la obtención de la información se realiza a través de fuentes formales, como ser los medios de comunicación, libros, revistas, bases de datos, y de fuentes informales como competidores, clientes, proveedores, personal interno, congresos, ferias, y demás. La información obtenida se ha de analizar, procesar y distribuir a las personas adecuadas. Por lo tanto, como Equipo avocado al estudio de la disciplina de VT, hemos considerado sumamente oportuno y necesario diseñar y desarrollar un Sistema de VT, cuyo objetivo troncal es brindar alertas con respecto a cambios que se estimen importantes para la organización y colaborar en la búsqueda de información útil que implique una valiosa contribución para el proceso de toma de decisiones.

Es difícil o casi imposible estar informado de todo permanentemente, por lo tanto, la empresa debe definir sus requerimientos de información. Los aspectos determinantes para la continuidad de la empresa se denominan "factores críticos" y se definen varios ámbitos: mercado, tecnología, proveedores y competidores. Este es el motor impulsor que nos motivó a desarrollar un prototipo de un Sistema de VT en el ámbito de la Universidad Tecnológica Nacional- Facultad Regional Tucumán.

El Sistema de VT se inviste de trascendental relevancia ya que contribuye a optimizar la planificación estratégica, permite aumentar el conocimiento del entorno, detectar oportunidades y amenazas, afianzando los criterios que intervienen siempre en toda toma de decisiones. Nuestro desarrollo nos permitió identificar y abordar las cuestiones fundamentales de todo proceso de vigilancia, obteniendo de ello la definición de las siguientes características con las que hemos dotado al Sistema de VT:

- Detectar cambios y anticiparse en aspectos como nuevas tecnologías, nuevos productos, nuevos competidores, nuevos bienes de equipo.

- Mejorar el conocimiento de la propia UTN - FRT (capacidades tecnológicas, relaciones de dependencia entre tecnologías / normas / productos, debilidades y fortalezas con respecto a clientes, proveedores).

- Obtener información sobre nuevas amenazas para reducir riesgos, que pueden venir en forma de nuevas patentes, nuevas reglamentaciones, alianzas estratégicas, entre otros.

- Permitir identificar a posibles socios para cooperar, detectar nuevos productos o desarrollos de interés, redes de colaboración y demás.

Es necesario mencionar que nuestro Sistema de VT actualmente se encuentra en fase de prueba dentro del ámbito de la comunidad de la UTN - FRT, siendo por el momento un prototipo funcional que está en un constante proceso de 
retroalimentación que nos permite poner ojo clínico en detalles que consideramos importantes, para que en un futuro cercano se convierta en un poderoso recurso de software capaz de aplicarse en cualquier organización. Sin embargo, estamos en condiciones de aseverar que nuestro sistema contribuye a la obtención de los siguientes beneficios:

- Mejora el proceso de innovación.

- Mejora las condiciones necesarias para encarar un proceso de cambio.

- Permite tomar decisiones en base a consideraciones objetivas de innovación.

- Mejora en la estructura curricular de las materias, mediante un constante proceso de vigilancia.

- Permite ampliar el espectro de visión sobre las tendencias tecnológicas.

Insistimos nuevamente y hacemos puntual hincapié en la necesidad de contar con un Sistema de VT ya que, a nivel tecnológico, las innovaciones se disparan constantemente y esto representa cierta dificultad para estar informado de las últimas novedades y conocer hacia dónde se decantan las mejores soluciones. La globalización ha supuesto un desbordamiento en el volumen de información disponible, siendo frecuente identificar numerosas empresas que realizan inversiones en desarrollos para los cuales ya existe una solución, lo que implica grandes pérdidas económicas. Y contar con un software que lleve a cabo una profunda tarea de vigilancia teniendo en cuenta las necesidades de la organización que la aplique, se interpreta como una herramienta vinculada directamente con la Gestión del Conocimiento en cuanto a las áreas de I + D + i se refiere; un sistema capaz de identificar, capturar y plasmar aquella información considerada crítica y vital para la supervivencia y éxito de toda organización comprometida con la innovación.

\section{Conclusión}

La Vigilancia Tecnológica revela que existen pocas capacidades de investigación y desarrollo que fortalezcan las competencias e innovaciones, es por ello que se hace imperativo buscar mecanismos que permitan el fortalecimiento de éstas capacidades que puede darse por las siguientes vías:

- Formación de investigadores en países líderes en investigación y desarrollo.

- Seguimiento de tecnologías de procesos y productos desarrollados.

La VT es un mecanismo esencial para facilitar las decisiones estratégicas de una organización, que pueden incluir perfectamente la creación de una línea de I $+\mathrm{D}+\mathrm{i}$ dentro de la empresa para investigar sobre cierta tecnología.

Las fuentes de información son esenciales en la VT. Ésta, consiste en captar información del entorno, seleccionar la que se considere relevante para el negocio, hacer un seguimiento de la evolución de las tecnologías claves de cada sector y 
difundir toda esa información en la empresa para su utilización como herramienta para la toma de decisiones, de manera que podamos anticiparnos a los cambios que se van a producir.

En definitiva, la VT es un proceso muy útil para filtrar y sintetizar el aluvión de información bruta que caracteriza la era de Internet. Las empresas tienen necesidad de conocer lo que se está haciendo en cada campo de su interés y por otro lado se ven saturadas de información (relevante e irrelevante) que debe ser organizada.

A lo largo de la presente ponencia, pusimos énfasis en manifestar que la VT y las herramientas de software se encuentran unidas por un fuerte lazo que logra amalgamarlas en un eficaz complemento que lo transforman en un binomio irreducible. Un Sistema de VT tiene la finalidad de servir como elemento facilitador en el proceso de Vigilancia Tecnológica, reduciendo complicaciones innecesarias y dotando a la organización que lo implemente de dinamismo y versatilidad. Esto se traduce en beneficios económicos que significan un ahorro que las empresas realizan al tomar la información existente y no producirla en forma redundante.

\section{Referencias}

Palop, F.; Vicente, J. M. (1999). Vigilancia Tecnológica e Inteligencia competitiva. Su potencial para la empresa española. COTEC, Madrid.

Escorsa, P.; Maspons, R. (2001) De la vigilancia tecnológica a la inteligencia competitiva. Prentice Hall, Madrid.

León López, A.; Castellanos Domínguez, O.; Montañez Franco, V. (2008). Tendencias actuales en el entendimiento de la Vigilancia Tecnológica como instrumento de inteligencia en la organización. COTEC, Madrid.

Colombia, Ministerio de Agricultura y Desarrollo Rural (2008). Estudios de Vigilancia Tecnológica aplicados a cadenas productivas del sector agropecuario colombiano. Giro Editores Ltda., Bogotá.

Villanueva, Martin (2010). Herramienta clave para Estructuras de Apoyo al desarrollo de Procesos Emprendedores: Vigilancia Tecnológica e Inteligencia Competitiva. Ponencia presentada en el 8th World Congress of Engineering Education, Buenos Aires, Argentina. 